\section{References}

1. Li Q, Guan X, Wu P, et al. Early transmission dynamics in Wuhan, China, of novel coronavirus-infected pneumonia. N Engl J Med 2020;382:1199-1207.

2. Pan Y, Zhang D, Yang P, Poon LLM, Wang Q. Viral load of SARS-CoV-2 in clinical samples. Lancet Infect Dis 2020;20:411-412.
3. Liu Y, Yan LM, Wan L, et al. Viral dynamics in mild and severe cases of COVID-19. Lancet Infect Dis March 19, 2020 [Epub ahead of print]. doi: 10.1016/S1473-3099(20)30232-2.

\title{
Critical role of Wuhan cabin hospitals in controlling the local COVID-19 pandemic
}

\author{
Wenlong Yao MD, Xueren Wang MD, PhD and Tianzhu Liu MD (1) \\ Department of Anesthesiology, Tongji Hospital, Tongji Medical College, Huazhong University of Science and Technology, Wuhan, China
}

To the Editor-COVID-19 is quickly spreading all over the world. The total number of confirmed cases has exceeded 1.6 million in just 2 months. ${ }^{1}$ Patients with a variety of respiratory symptoms have flooded into hospitals in a relative short time, posing an enormous challenge to every healthcare system. Wuhan was the first center of the pandemic, and it had the highest number of cases in China. But the pandemic in Wuhan was controlled by 2 months of lockdown beginning January 23, 2020, and newly detected cases of COVID-19 have now decreased to zero. Among a series of preventive approaches, ${ }^{2}$ cabin hospitals played a critical role in isolating mild and asymptomatic cases. Here, we evaluate the role of cabin hospitals in controlling the COVID-19 pandemic by retrospectively analyzing the correlation between available beds in cabin hospitals and epidemic data.

We obtained the data regarding total daily beds available in cabin hospitals from the official website of the Wuhan municipal government, and we extracted daily numbers of newly diagnosed cases, newly cured cases, and new deaths, and we calculated the overall recovery rate and mortality from COVID-19 in Wuhan from the official website of the National Health Commission of the People's Republic of China. COVID-19 cases were diagnosed according to history, symptoms, chest CT, and nucleic acid test. ${ }^{3}$ From February 12 to February 14, a clinical diagnosis of COVID-19 was applied to make sure that every patient received immediate treatment in Wuhan. Therefore, the number of cases diagnosed in these 3 days dramatically increased, and we was excluded these data from our analysis. We used SPSS version 19.0 software (IBM, Armonk, NY) for the statistical analysis. A Pearson correlation analysis was performed by correlating cabin beds with all epidemic data. $P<.05$ was considered a significant difference.

The official government website reported a total of 28 designated hospitals with 8,254 beds for COVID-19 patients in Wuhan before February 4, 2020. The utilization ratio of beds was as high as $99.1 \%$. On February 4, 2020, the first cabin hospital in Hongshan stadium opened with 1,000 beds. By February 26, 2020, a total of 17 cabin hospitals with 35,499 beds had been set up in Wuhan; overall these cabin hospitals received $\sim 12,000$ mild cases of COVID-19. The final utilization ratio of cabin beds was

Author for correspondence: Tianzhu Liu, Department of Anesthesiology, Tongji Hospital, Tongji Medical College, Huazhong University of Science and Technology, Wuhan, China. E-mail: liutzh@126.com

Cite this article: Yao W, Wang X, and Liu T. (2020). Critical role of Wuhan cabin hospitals in controlling the local COVID-19 pandemic. Infection Control \& Hospital Epidemiology, 41: 1356-1358, https://doi.org/10.1017/ice.2020.167 $\sim 33.8 \%$. All epidemiological data and their fluctuating trends with the increase in cabin beds are shown in Figure 1.

By statistical analysis, the number of newly diagnosed cases showed a highly negative correlation with the availability of cabin beds $(\mathrm{r}=-0.833 ; P<.0001)$. We detected a highly negative correlation between the number of new death cases and the number of cabin beds $(\mathrm{r}=-0.859 ; P<.0001)$. The overall recovery rate was positive correlated with cabin beds $(\mathrm{r}=0.961 ; P<.0001)$. In addition, we detected a significantly decrease of severe cases in the hospital with the increase of cabin beds $(\mathrm{r}=-0.977 ; P<.0001)$. [A correlation coefficient of $0.8-1.0$ indicates a high correlation; 0.6-0.8 indicates a strong correlation; $0.4-0.6$ indicates a moderate correlation; $0.2-0.4$ indicates a weak correlation; and 0.0-0.2 indicates a very weak or no correlation.]

The approaches for prevention and control of COVID-19 can vary from city to city. However, the principle of controlling contagious diseases is to isolate the source of infection, to cut off transmission, and to protect vulnerable populations. ${ }^{4}$ Although both COVID-19 and SARS are respiratory diseases caused by coronavirus, COVID-19 differs from SARS ${ }^{5}$ in that many mild and asymptomatic cases of COVID-19 also have transmissibility, and these cases are often missed and not isolated. Therefore, the management of mild or asymptomatic COVID-19 cases is equally important as the treatment of severe cases. Our analysis showed that, with the increase of available beds by cabin hospitals, the newly diagnosed cases and severe cases decreased. Thus, the cabin hospitals played an important role in controlling the COVID-19 pandemic. They effectively prevented family infection or community spread. Early treatment of mild cases can prevent COVID-19 cases from deteriorating.

Cabin hospitals were mainly responsible for the treatment of mildly ill patients. All admitted patients were diagnosed by a positive nucleic acid test, concern regarding cross infection was alleviated. In these temporary hospitals, patients were also cared for by professional medical staff. When a case became severe, the patient was transferred to a designated infectious hospital immediately. Food, accommodation, medication, and examination were paid by the government. These incentives greatly increased the motivation of mildly ill patients to be admitted to cabin hospitals, which reduced social mobility and the risk of community infection. At the same time, timely medical treatment also improved prognoses, avoiding exacerbation of the disease. ${ }^{6}$ In addition, initiation of cabin hospitals reduced the workload of designated infectious hospitals, so the

(c) 2020 by The Society for Healthcare Epidemiology of America. All rights reserved. This is an Open Access article, distributed under the terms of the Creative Commons Attribution licence (http://creativecommons.org/licenses/by/4.0/), which permits unrestricted re-use, distribution, and reproduction in any medium, provided the original work is properly cited. 

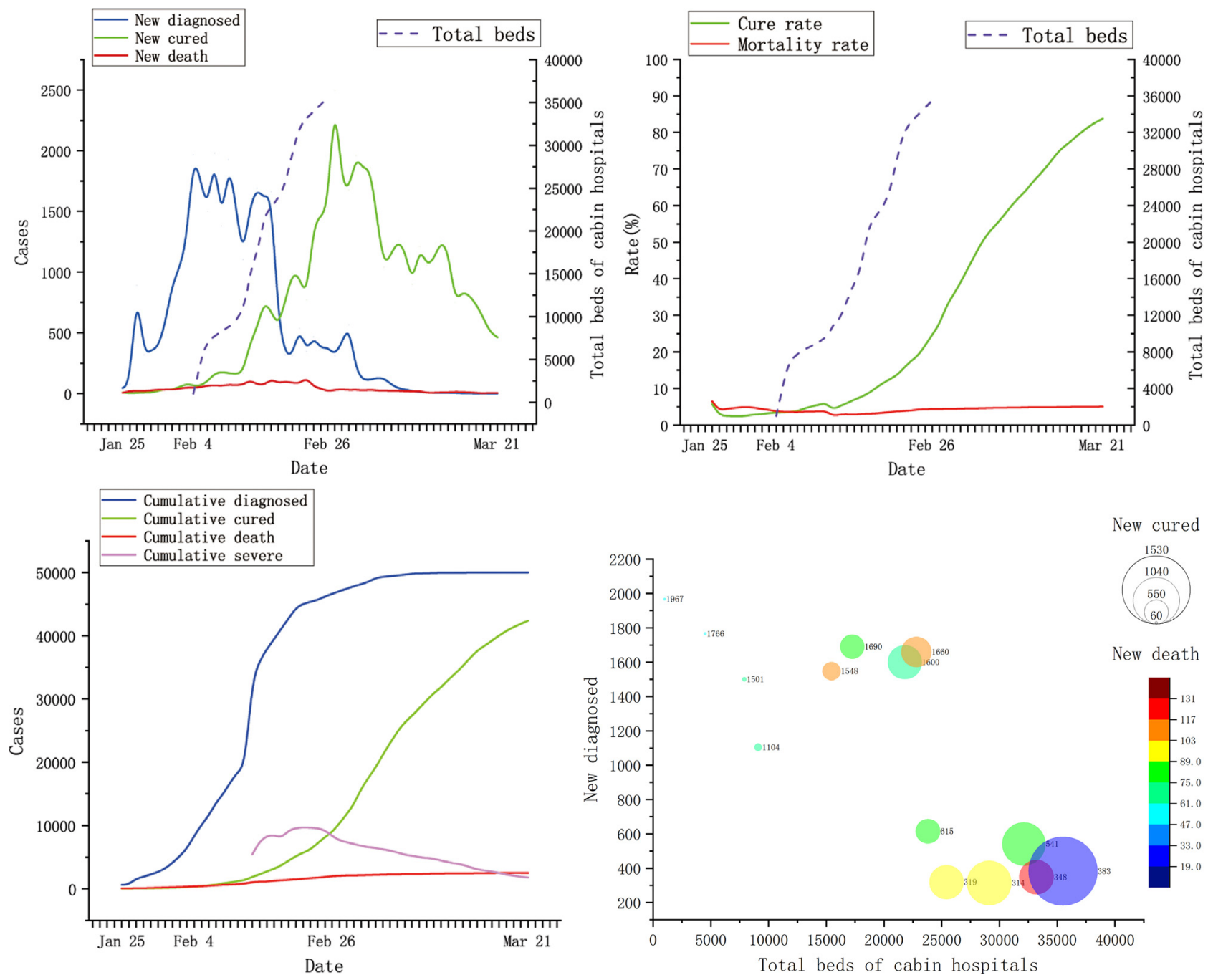

Fig. 1. The relationships between total beds of cabin hospitals and epidemic data of COVID-19 in Wuhan. Data were obtained from National Health Commission of China and people's government of Wuhan to Mar 22, 2020.

limited public medical resources could be used to treat severe patients and thus reduce the death rate.

According to $\mathrm{Xu}$ et $\mathrm{al},{ }^{7}$ the cost of cabin hospitals was low enough that the government could support the roll out on a large enough scale to ensure rapid sequester of cases. Short-term training should be employed to equip cabin hospital staff with self-protection and medical care. Psychological counseling for patients and medical staff should be provided to alleviate anxiety and panic. We also advocate communication and entertainment activity between patients. Online visits for comprehensive mental consultation were also available.

A cabin hospital is like a large community clinic. Home quarantine and community isolation play an important role in the treatment of mild cases, but there is a risk of neglecting some cases, which could lead to community transmission, and a percentage of patients become severely ill. In Wuhan, cabin hospitals connected traditional community clinics and hospitals to achieve early diagnosis, timely treatment, and effective isolation of COVID-19 patients. In conclusion, these cabin hospitals were an important part of effectively controlling the COVID-19 pandemic in Wuhan.

Supplementary material. To view supplementary material for this article, please visit https://doi.org/10.1017/ice.2020.167
Acknowledgments. None.

Financial support. No financial support was provided relevant to this article.

Conflicts of interest. All authors report no conflicts of interest relevant to this article.

\section{References}

1. Coronavirus disease (COVID-2019) situation reports. World Health Organization website. https://www.who.int/docs/default-source/coronaviruse/ situation-reports/20200411-sitrep-82-covid-19.pdf?sfvrsn=74a5d15_2. Published 2020. Accessed April 12, 2020.

2. Pan A, Liu L, Wang C, et al. Association of public health interventions with the epidemiology of the COVID-19 outbreak in Wuhan, China. JAMA 2020 Apr 10 [Epub ahead of print]. doi: 10.1001/jama.2020.6130.

3. Zhou F, Yu T, Du R, et al. Clinical course and risk factors for mortality of adult inpatients with COVID-19 in Wuhan, China: a retrospective cohort study. Lancet 2020;395:1054-1062.

4. Cheng PK, Wong DA, Tong LK, et al. Viral shedding patterns of coronavirus in patients with probable severe acute respiratory syndrome. Lancet 2004;363:1699-1700.

5. Wilder-Smith A, Chiew CJ, Lee VJ. Can we contain the COVID-19 outbreak with the same measures as for SARS? Lancet Infect Dis 2020 March 5 [Epub ahead of print]. doi: 10.1016/S1473-3099(20) 30129-8. 
6. Liu Y, Yan LM, Wan L, et al. Viral dynamics in mild and severe cases of COVID-19. The Lancet Infectious diseases 2020 March 19 [Epub ahead of print]. doi: 10.1016/S1473-3099(20)30232-2.
7. Zhu W, Wang Y, Xiao K, et al. Establishing and managing a temporary coronavirus disease 2019 specialty hospital in Wuhan, China. Anesthesiology 2020 March 19 [Epub ahead of print]. doi: 10.1097/ALN.0000000000003299.

\title{
Diagnostic options for coronavirus disease 2019 (COVID-19)
}

\author{
Yuanyuan Xiao $\mathrm{MM}^{1}$ (10), Zhong Peng $\mathrm{MD}^{2}$, Caixia Tan $\mathrm{MM}^{1}$, Xiujuan Meng $\mathrm{MD}^{1}$, Xun Huang $\mathrm{MD}^{1,3}$, Anhua $\mathrm{Wu}^{1,3}$ and \\ Chunhui Li MD ${ }^{1,3}$ (D) \\ ${ }^{1}$ Xiangya Hospital Central South University, Changsha, Hunan Province, China, ${ }^{2}$ State Key Laboratory of Agricultural Microbiology, College of Veterinary Medicine, \\ Huazhong Agricultural University, Wuhan 430070, Hubei, China and ${ }^{3}$ National Clinical Research Center for Geriatric Disorders (Xiangya Hospital), Changsha, \\ Hunan Province, China
}

To the Editor-The COVID-19 pandemic is posing a great challenge to global health and economy. Early accurate diagnosis plays a key role in fighting the disease. However, the diagnosis might be missed because of false-negative tests due to the insufficient sensitivity of the only test that detects SARS-CoV-2 or coinfection with other viruses. These false-negative results will affect clinical management decisions as well as control of the epidemic. Therefore, broader viral tests should be given to patients suspected to have COVID-19. Here, we discuss the existing methods of diagnosing COVID-19.

\section{Nucleic acid amplification tests (NAAT)}

The current first choice for the etiological diagnosis of COVID-19 is based on detection of unique sequences of virus RNA by real-time reverse-transcription polymerase chain reaction (rRT-PCR). ${ }^{1}$ The PCR test is appropriate for the acute phase of illness; however, cases of missed diagnoses have already been reported using this method. ${ }^{2,3}$ Recently, related research shows that the COVID-19-RdRp/Hel rRT-PCR test is highly sensitive and specific, which might help to reduce the false-negative rate and would be significantly useful for detecting specimens with low viral loads. ${ }^{3}$ Thus, in terms of technical and financial support, the current rRT-PCR testing available is relatively optimal for SARS-CoV-2 screening of suspected cases.

\section{Viral sequencing}

The application of next-generation sequencing may be an accurate diagnosis method for SARS-CoV-2, including metagenomics, hybrid capture-based sequencing, and amplicon-based next-generation sequencing. ${ }^{1,45}$ These 3 approaches show a higher sensitivity than conventional RT-PCR, and they can meet the need for secondary detection, diagnosis confirmation, and large-scale detection of RT-PCR false-negative results. ${ }^{5}$ However, high cost is currently an important obstacle to more widespread use of virus sequencing.

\section{Serological testing}

For patients with COVID-19, detectable SARS-CoV-2 antibodies are mainly divided into IgM and IgG. In general, most of SARS-CoV-2-specific IgM antibodies can be detected 3-5 days after

\footnotetext{
Authors for correspondence: Chunhui Li, Email: lichunhui@csu.edu.cn. Or Anhua Wu, Email: xywuanhua@csu.edu.cn

Cite this article: Xiao Y, et al. (2020). Diagnostic options for coronavirus disease 2019 (COVID-19). Infection Control \& Hospital Epidemiology, 41: 1358-1359, https://doi.org/ 10.1017/ice.2020.168
}

onset, and during the recovery period, IgG antibody titers are $\geq 4$ times higher than in the acute phase. ${ }^{4,6}$ An antibody test is appropriate for the convalescence phase of COVID-19 in case of a symptomatic infection. This method, however, is susceptible to the presence of some interfering substances in the blood sample (eg, rheumatoid factor, nonspecific IgM, etc), and therefore, it has a very high false-positive rate. Hence, SARS-CoV-2-specific IgM or IgG antibody testing can be used as a diagnostic standard for COVID-19 in the case of a negative NAAT, when 2 dynamic tests are required. ${ }^{1,6}$

\section{Rapid antigen tests}

In theory, rapid antigen tests have the advantages of fast detection speed and low cost, but as yet they have poor sensitivity and specificity for detecting coronaviruses (except MERS). ${ }^{7}$ Moreover, it is almost impossible to identify patients in the incubation period of infection, which is to say that antigen tests cannot be used as the sole basis for the diagnosis or exclusion of COVID-19. A pre-peerreviewed article reported that a fluorescence immunochromatographic assay is an accurate, rapid, early and simple method for detecting the nucleocapsid protein of SARS-CoV-2 in nasopharyngeal swab samples and urine samples for the diagnosis of COVID-19. ${ }^{8}$ This claim requires further investigation.

\section{Imaging examinations}

Because lung abnormalities may appear ahead of clinical manifestations and positive NAAT, some studies have recommended that early chest computerized tomography (CT) be used to screen suspected cases of COVID-19., 2,9,10 Furthermore, pneumonia manifests with chest CT imaging and suggests the evolution and prognosis of COVID-19. ${ }^{2,10}$ Nevertheless, due to the highly contagious nature of SARS-CoV-2 and the risk of transporting critically ill patients, the choice to conduct a chest CT scan in patients with suspected or established COVID-19 is made infrequently. In addition, lung ultrasonography may have great utility in managing COVID-19 pneumonia due to its safety, repeatability, absence of radiation, low cost, and point-ofcare use. ${ }^{9}$ For cases in which pulmonary ultrasound is not sufficient to answer clinical questions, a chest CT is needed.

In summary, combining assessment of imaging features with clinical and laboratory findings could facilitate early diagnosis of COVID-19. Here, we have systematically summarized the various diagnostic methods for SARS-CoV-2. More importantly, this work offers practical options for diagnosing COVID-19. Our experience

(C) 2020 by The Society for Healthcare Epidemiology of America. All rights reserved. This is an Open Access article, distributed under the terms of the Creative Commons Attribution licence (http://creativecommons.org/licenses/by/4.0/), which permits unrestricted re-use, distribution, and reproduction in any medium, provided the original work is properly cited. 